\title{
Effect of fractional ablative carbon dioxide laser with lidocaine spray on skin flap survival in rats
}

\author{
Manki Choi ${ }^{1}$, \\ Youngsoo Park', \\ Yong-Ha Kim ${ }^{1}$, \\ Kyu Jin Chung ${ }^{1}$ \\ ${ }^{1}$ Department of Plastic and \\ Reconstructive Surgery, Yeungnam \\ University College of Medicine, Daegu; \\ ${ }^{2}$ Iwell Plastic Surgery, Seoul, Korea
}

\begin{abstract}
Background: Lidocaine spray is a local anesthetic that improves random-pattern skin flap survival. The fractional ablative carbon dioxide laser (FxCL) produces vertical microchannels that delivers topically applied drugs to the skin. In this study, we hypothesized that FxCL therapy would enhance the lidocaine effect to improve random-pattern skin flap survival in rats.

Methods: McFarlane random-pattern skin flaps were elevated in 48 rats, which were divided into four groups according to treatment: FxCL+lidocaine, FxCL, lidocaine, and nontreatment (control). On postoperative day 7 , necrotic flap areas, the number of capillary vessels, and neutrophil count were evaluated. Anti-rat vascular endothelial growth factor (VEGF) and CD31 antibody activity were also evaluated by immunohistochemical staining.

Results: Flap survival rate was $53.41 \% \pm 5.43 \%, 58.16 \% \pm 4.80 \%, 57.08 \% \pm 5.91 \%$, and $69.08 \% \pm$ $3.20 \%$ in the control, lidocaine, FxCL, and FxCL+lidocaine groups, respectively. Mean neutrophil count in the intermediate zone excluding the necrotic tissue was $41.70 \pm 8.40,35.43 \pm 6.41,37.23 \pm$ 7.15 , and $27.20 \pm 4.24$ cells/field in the control, lidocaine, FxCL, and FxCL+lidocaine groups, respectively. Anti-rat VEGF and CD31 antibody activity were the highest in the FxCL+lidocaine group. Conclusion: FxCL with lidocaine had a positive effect on random-pattern skin flap survival in rats. Thus, FxCL with lidocaine spray should be considered as a new treatment option to improve flap viability.
\end{abstract}

Keywords: Lasers, gas / Lidocaine / Rats

\section{INTRODUCTION}

Local random-pattern skin flaps are commonly used to reconstruct soft tissue defects. However, flap necrosis remains a difficult challenge for the plastic surgeon. Blood supply deficiency and ischemic-reperfusion injury are generally responsible for flap necrosis [1].

Various trials have been conducted to reduce distal flap necro-

\section{Correspondence: Yong-Ha Kim}

Department of Plastic and Reconstructive Surgery, Yeungnam University College

of Medicine, 170 Hyeonchung-ro, Nam-gu, Daegu 42415, Korea

E-mail: yhkim@med.yu.ac.kr

This study was funded by the 2016 Yeungnam University Research Grants.

Received July 29, 2019 / Revised August 16, 2019 / Accepted August 18, 2019 sis and achieve optimal results [2-6]. Lidocaine is an effective pharmacologic agent to increase flap survival $[7,8]$. It is a widely used amide-type anesthetic that blocks voltage-gated sodium channels in cell membranes of neurons and has been shown to improve cutaneous blood flow, inhibit platelet aggregation, and suppress neutrophil function [9-11]. Lidocaine is effective in recovery after flap reperfusion [12] and can improve randompattern skin flap survival [8]. To minimize unwanted adverse effects associated with systemic application and maintain a positive effect on the flap, lidocaine should be used topically.

The fractional ablative laser was introduced as a new technology for drug delivery enhancement [13]. Many studies have demonstrated that the laser can assist in topical drug delivery 
into the skin. Various compounds, including topical 5-aminolevulinic acid, methyl 5-aminolevulinate, 5-fluorouracil, ascorbic acid, corticosteroids, diclofenac, imiquimod, methotrexate, allogeneic stem cells, autologous platelet-rich plasma, and vaccines, have been investigated [14-18]. Lidocaine is a topical agent that demonstrated enhanced transdermal absorption in a fractional ablative laser in vivo model. Oni et al. [18] reported that the amount of local lidocaine uptake can be manipulated by changing the energy setting and ablative depth of the laser. In other words, the possibility of systemic adverse effects of lidocaine can be minimized by controlling the energy of the laser.

We hypothesized that fractional ablative laser therapy would enhance the lidocaine effect to improve random-pattern skin flap survival with minimal systemic adverse effects. Therefore, in this study, we investigated the effect of fractional ablative carbon dioxide laser (FxCL) and topical lidocaine spray on random-pattern skin flap survival in rats.

\section{METHODS}

\section{Animals and groups}

The experimental protocol, including the use of animals in the research, was approved by the Institutional Animal Care and Use Committee of Yeungnam University. Forty-eight 2-monthold male Sprague-Dawley rats (weight, 200-250 g) were randomly divided into four groups according to treatment: FxCL+lidocaine $(\mathrm{n}=12), \operatorname{FxCL}(\mathrm{n}=12)$, lidocaine $(\mathrm{n}=12)$, and nontreatment (control; $n=12$ ). All animals were provided with standard laboratory food and water ad libitum.

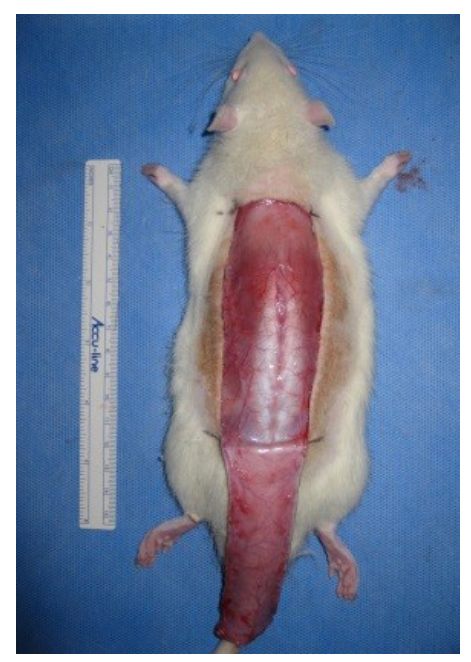

Fig. 1. Elevation of random-pattern skin flap in this study. Caudally pedicled McFarlane-type $3 \times 9-\mathrm{cm}$ random patterns skin flaps were made on the dorsal skin.

\section{Surgical procedure}

The surgical procedure was performed under general anesthesia via the intramuscular injection of zolazepam $(50 \mathrm{mg} / \mathrm{kg}$, Zoletil; Virbac, Carros, France) and xylazine (5 mg/kg, Rompun; Bayer Korea, Seoul, Korea). To prevent infectious complications, a single dose of cefazolin (60 mg/kg; Yuhan Co., Seoul, Korea) was administered before the procedure and an aseptic technique was rigorously applied during the procedure. After the dorsal skin was shaved with an electric shaver, a $3 \times 9-\mathrm{cm}$ caudally pedicled random-pattern skin flap, called a McFarlane flap, was designed on the skin. The palpable hip joints were used as anatomical landmarks to define the base of the flap. The incision was made, and the entire flap was undermined below the level of the panniculus carnosus. Then, the flap was completely separated from the deep fascia up to its base. After controlling any bleeding, a $3 \times 9-\mathrm{cm}$ silicone sheet was inserted between the muscle layer and skin flap to prevent muscle neovascularization and then immediately sutured back to the donor bed using 4-0 Nylon sutures (Fig. 1). All procedures were performed by one researcher (YP), and no rats died during the procedure. The flap area was equally divided into three distinct zones: proximal, intermediate, and distal (Fig. 2).

\section{Laser assembly}

FxCL (Edge ONE 2.0; Jeisys Medical Inc., Seoul, Korea) with a wavelength of $10,600 \mathrm{~nm}$ was used. The dimension of the treatment area of the handpiece was $10 \times 10 \mathrm{~mm}^{2}$. The fractional ablative laser total fluence energy setting used was $3.0 \mathrm{~J} / \mathrm{cm}^{2}$ with $120-\mu \mathrm{m}$ ablative depth. A probe setup with 100 dots of $1-\mathrm{cm}^{2}$ size was used to deliver laser energy to the skin flaps.

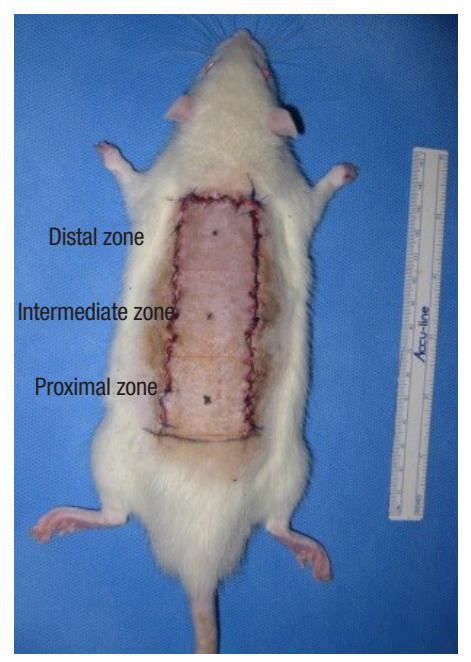

Fig. 2. Sutured random-pattern skin flap back to the donor bed. The sutured dorsal skin flap area was divided into three distinct zones equally $(3 \times 3 \mathrm{~cm})$ : proximal, intermediate, and distal zones. 


\section{Flap treatment in the four groups}

Following the surgical procedure, rats in the FxCL+lidocaine and FxCL groups received one pass of FxCL without missed or overlapped areas. Immediately following FxCL treatment, topical lidocaine spray (Xylocaine 10\% spray; AstraZeneca, Cambridge, England) was applied in the FxCL+lidocaine and lidocaine groups. The lidocaine concentration of topical lidocaine spray is $0.1 \mathrm{~g} / \mathrm{mL}$. From approximately $5 \mathrm{~cm}$ above the randompattern skin flap, the researchers perpendicularly sprayed the lidocaine on each proximal, intermediate, and distal zone of the flap. Lidocaine spray was sprayed once in each zone of the flap. The amount of lidocaine spray sprayed at one time was about $0.1 \mathrm{~mL}$. To prevent the lidocaine from penetrating the skin barrier through the incision site, the incision line was covered with sterile gauze. In the FxCL and control groups, $0.9 \%$ saline was applied to the skin flaps with the same pump of the topical lidocaine spray. Then, all rats were housed individually in standard experimental cages in an environmentally controlled room and were fed standard rat chow and water ad libitum for 7 days.

\section{Assessment of flap survival}

To quantify the survival area, all flaps were photographed with a digital camera (EOS 600D; Canon, Tokyo, Japan) and the survival area was measured using an image program (ImageJ software; National Institutes of Health). Results were expressed as percentage survival in relation to total surface area of the flap.

\section{Neutrophil and capillary vessel density}

A $1 \times 1-\mathrm{cm}$ skin flap around the demarcation line of the necrotic area was harvested including necrotic and survival site samples. All tissue specimens were fixed in $10 \%$ neutral buffered formalin for at least 24 hours at room temperature, embedded in paraffin, cut into 4-mm slices, and stained with hematoxylin and eosin $(\mathrm{H} \& \mathrm{E})$. Another $1 \times 1-\mathrm{cm}$ full-thickness tissue sample in the intermediate zone excluding necrotic tissue was harvested for neutrophil count under a light microscope $(\times 100)$. The number of erythrocyte-containing capillary vessels was also counted. These counts were performed in five areas under high magnification $(\times 200)$ in each area to calculate the capillary vessel density per $1 \mathrm{~mm}^{2}$. The blood vessels were measured at the level of the papillary dermis inferior to the panniculus carnosus.

\section{Immunohistochemical evaluation}

Paraffin-embedded $4-\mu \mathrm{m}$ sectioned tissue samples were immunostained with monoclonal antibody against rat vascular endothelial growth factor (VEGF; 1:100 dilution, ab28364 Abcam; Santa Cruz Biotechnology, Santa Cruz, CA, USA) and CD31
(1:100 dilution; Novus Biologicals, Littleton, CO, USA). Then, the sections were counterstained with Mayer's hematoxylin and mounted. The amount of VEGF- and CD31-positive cells were quantified in five fields per specimen $(\times 400)$.

\section{Statistical analysis}

Data on percentage of flap survival in the photographic evaluation, number of capillary vessels and neutrophils in the histologic evaluation, and VEGF/CD31-positive microvascular density are expressed as mean \pm standard deviation. One-way analysis of variance with Bonferroni correction was conducted in each group. Statistical analysis was performed using SPSS version 23.0 (IBM Corp., Armonk, NY, USA) with $p$-values $<0.05$ considered statistically significant.

\section{RESULTS}

\section{Assessment of flap survival}

Percentage of survival area on postoperative day 7 was $69.08 \% \pm 3.20 \%$ in the FxCL+lidocaine group, $57.08 \% \pm 5.91 \%$ in the FxCL group, $58.16 \% \pm 4.80 \%$ in the lidocaine group, and $53.41 \% \pm 5.43 \%$ in the control group. Percentage of survival area in the FxCL+lidocaine group was significantly higher than that in the other three groups $(p<0.001)$. However, differences between the FxCL, lidocaine, and control groups with respect to percentage of survival area were not statistically significant $(p=0.18)$ (Table 1, Fig. 3).

\section{Neutrophil and capillary vessel density}

The stratum corneum of the random-pattern skin flap was successfully disrupted by the laser in the FxCL+lidocaine and FxCL groups (Fig. 4). Full-thickness tissue in the intermediate zone excluding the necrotic tissue presented differently under the light microscope on postoperative day 7. Dense neutrophil infiltration ( $41.70 \pm 8.40)$ was observed in the control group, whereas it was markedly decreased in the FxCL+lidocaine group $(27.20 \pm 4.24, p<0.001)$. Thus, the inflammatory reaction was less severe in the FxCL+lidocaine group than in the control group. There was no statistically significant difference in neutrophil count in the FxCL $(37.23 \pm 7.15)$ and lidocaine (35.43 \pm 6.41) groups compared with the control group (Fig. 5). Capillary vessel density (number of vessels in $1 \mathrm{~mm}^{2}$ ) in the dermal papillary layer was $17.74 \pm 5.38$ in the $\mathrm{FxCL}+$ lidocaine group, $18.51 \pm 3.57$ in the FxCL group, $16.10 \pm 6.04$ in the lidocaine group, and $18.14 \pm 4.88$ in the control group, without significant differences among the groups $(p=0.22)$. Capillary vessel density in the layer inferior to the panniculus carnosus was $13.91 \pm$ 4.98 in the FxCL+lidocaine group, $15.84 \pm 4.09$ in the FxCL 
Table 1. Percentage of survival area (\%) using photograph in four groups

\begin{tabular}{lcccc}
\hline Rat no. & $\begin{array}{c}\text { FxCL+lidocaine } \\
\text { group (\%) }\end{array}$ & $\begin{array}{c}\text { FxCL } \\
\text { group (\%) }\end{array}$ & $\begin{array}{c}\text { Lidocaine } \\
\text { group (\%) }\end{array}$ & $\begin{array}{c}\text { Control } \\
\text { group (\%) }\end{array}$ \\
\hline 1 & 74 & 63 & 54 & 53 \\
2 & 72 & 62 & 58 & 57 \\
3 & 69 & 61 & 57 & 58 \\
\hline 4 & 67 & 67 & 65 & 48 \\
\hline 5 & 66 & 52 & 62 & 46 \\
6 & 73 & 50 & 58 & 57 \\
7 & 64 & 48 & 54 & 52 \\
8 & 71 & 56 & 59 & 59 \\
9 & 69 & 52 & 50 & 48 \\
10 & 74 & 60 & 59 & 62 \\
11 & 68 & 54 & 67 & 46 \\
12 & 71 & 60 & 55 & 55 \\
Mean \pm SD (\%) & $69.08 \pm 3.20^{\text {a) }}$ & $57.08 \pm 5.91$ & $58.16 \pm 4.80$ & $53.41 \pm 5.43$ \\
\hline
\end{tabular}

FXCL, fractionated carbon dioxide laser.

a) $p<0.05$.

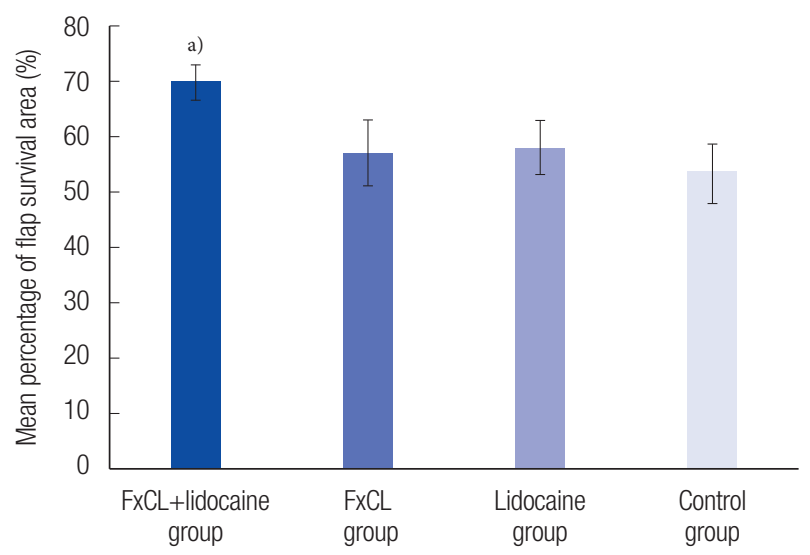

Fig. 3. Mean percentage of flap survival area (\%) using photographs. The results were $69.08 \% \pm 3.20 \%$ in the $\mathrm{FxCL}+$ lidocaine group, $57.08 \% \pm 5.91 \%$ in the FxCL group, $58.16 \% \pm 4.80 \%$ in the lidocaine group, and $53.41 \% \pm 5.43 \%$ in the control group (mean \pm SD). The flap survival in the FxCL+lidocaine group improved significantly. FxCL, fractional ablative carbon dioxide laser. ${ }^{a} p<0.05$.

group, $14.36 \pm 6.11$ in the lidocaine group, and $13.28 \pm 3.94$ in the control group. Similarly, there was no significant difference among the groups.

\section{Immunohistochemical evaluation}

Immunohistochemical evaluation revealed more VEGF-positive cells in the FxCL+lidocaine group than in the other three groups (FxCL+lidocaine, $121.64 \pm 19.38$ cells/field; FxCL, $92.45 \pm 16.11$ cells/field; lidocaine, $98.12 \pm 17.83$ cells/field; control, $86.09 \pm 12.51$ cells/field; $p<0.05$ ) (Figs. 6, 7). The number of CD31-positive microvascular vessels was also significantly higher in the FxCL+lidocaine group than in the other three groups

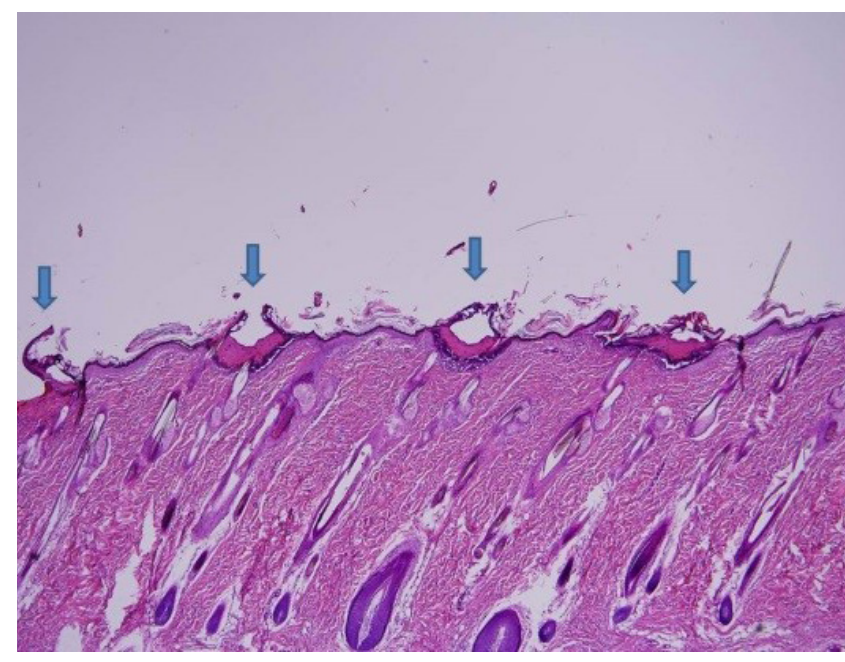

Fig. 4. Histologic findings $(\mathrm{H} \& \mathrm{E}, \times 40)$ of random-pattern skin flap in the FxCL+lidocaine group. Blue arrows indicate MAZs by FxCL. FxCL, fractional ablative carbon dioxide laser; MAZ, microscopic ablation zone.

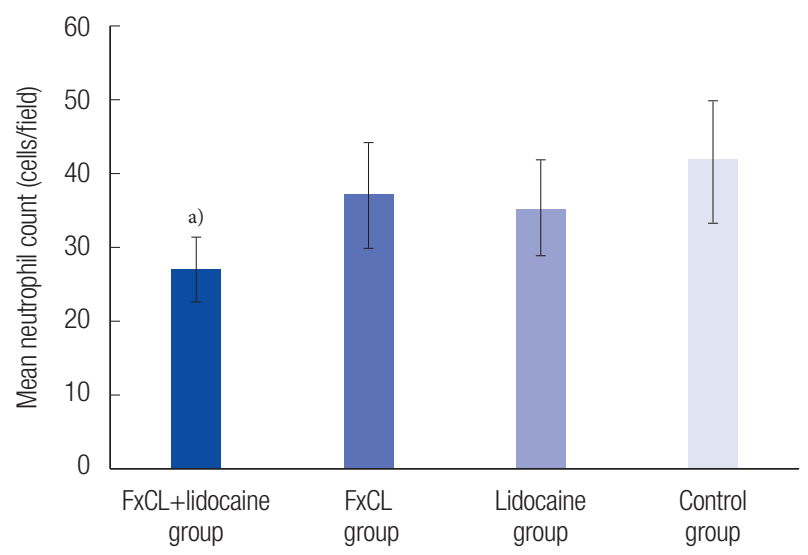

Fig. 5. Mean neutrophil count in the intermediate zone except necrotic tissue under high-power fields $(\times 200)$. The results were $27.20 \pm 4.24$ cells/field in the FxCL+lidocaine group, $37.23 \pm 7.15$ cells/field in the FxCL group, $35.43 \pm 6.41$ cells/field in the lidocaine group, and $41.70 \pm 8.40$ cells/field in the control group (mean $\pm S D)$. The mean neutrophil count in the FxCL+lidocaine group was significantly lower than that in other groups. FxCL, fractional ablative carbon dioxide laser. ${ }^{a)} p<0.05$.

(FxCL+lidocaine, $31.38 \pm 9.36$ vessels/field; FxCL, $22.52 \pm 3.99$ vessels/field; lidocaine, $24.66 \pm 8.25$ vessels/field; control, $18.45 \pm$ 5.89 vessels/field; $p<0.05$ ) (Figs. 8, 9).

\section{DISCUSSION}

The results of this study showed that flap survival was significantly higher in the FxCL+lidocaine group than that in the other groups. In addition, neutrophil count was significantly lower in the FxCL+lidocaine group than in the other groups, while neoangiogenetic parameters, VEGF and CD31, were signifi- 

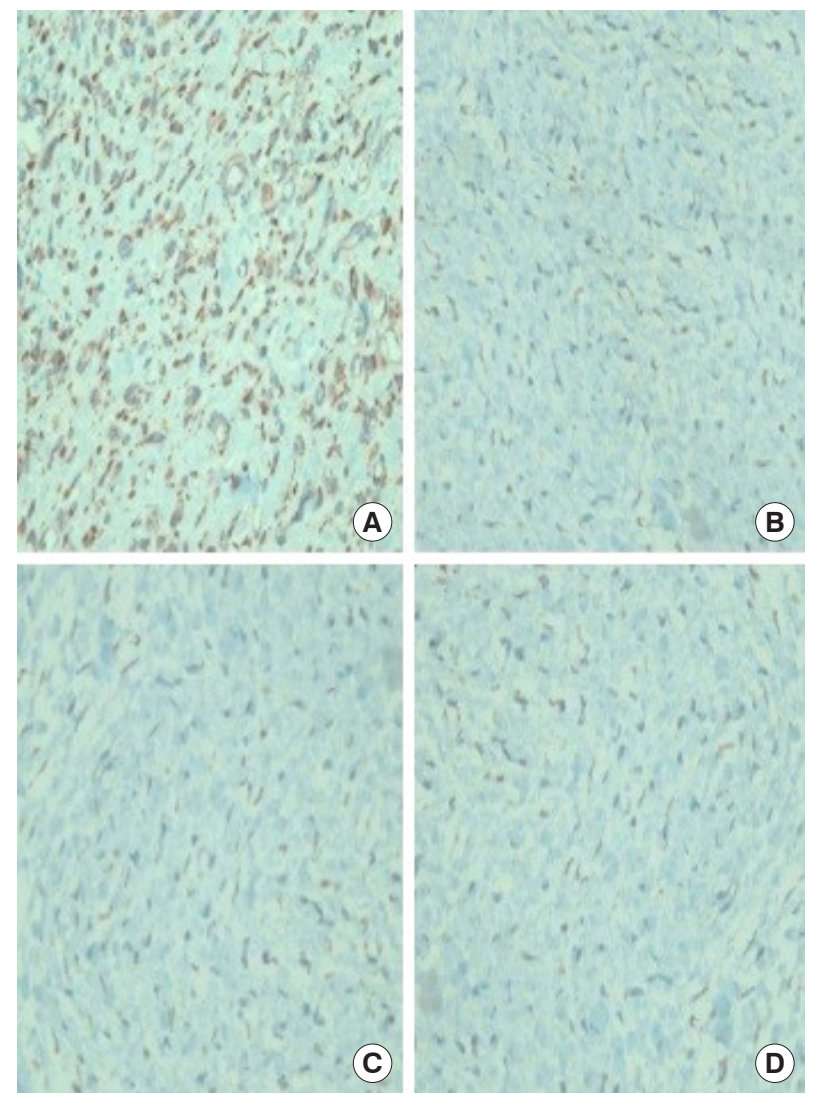

Fig. 6. Immunohistochemical staining of anti-rat VEGF antibody $(\times 400)$. The results showed more VEGF-positive staining cells (brown) in the FxCL+lidocaine group (A) than in the FxCL (B), lidocaine (C), and control groups (D). VEGF, vascular endothelial growth factor; FxCL, fractional ablative carbon dioxide laser.

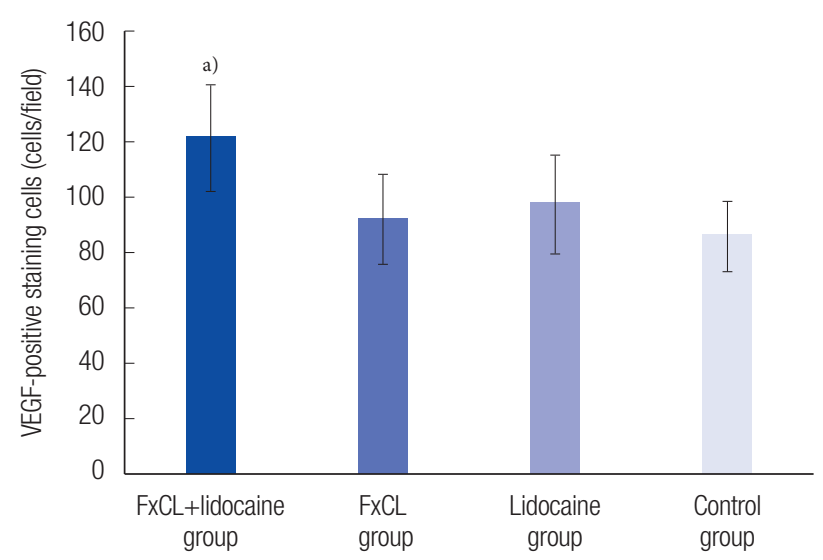

Fig. 7. VEGF-positive staining cells in the immunohistochemical evaluation. The number of VEGF-positive staining cells was significantly higher in the FxCL+lidocaine group than those in other groups (FxCL+lidocaine group, 121.64 \pm 19.38 cells/field; FxCL group, 92.45 \pm 16.11 cells/field; lidocaine group, $98.12 \pm 17.83$ cells/field; control group, $86.09 \pm 12.51$ cells/field). VEGF, vascular endothelial growth factor; FxCL, fractional ablative carbon dioxide laser. ${ }^{a} p<0.05$.

cantly higher in the FxCL+lidocaine group than in the other groups.
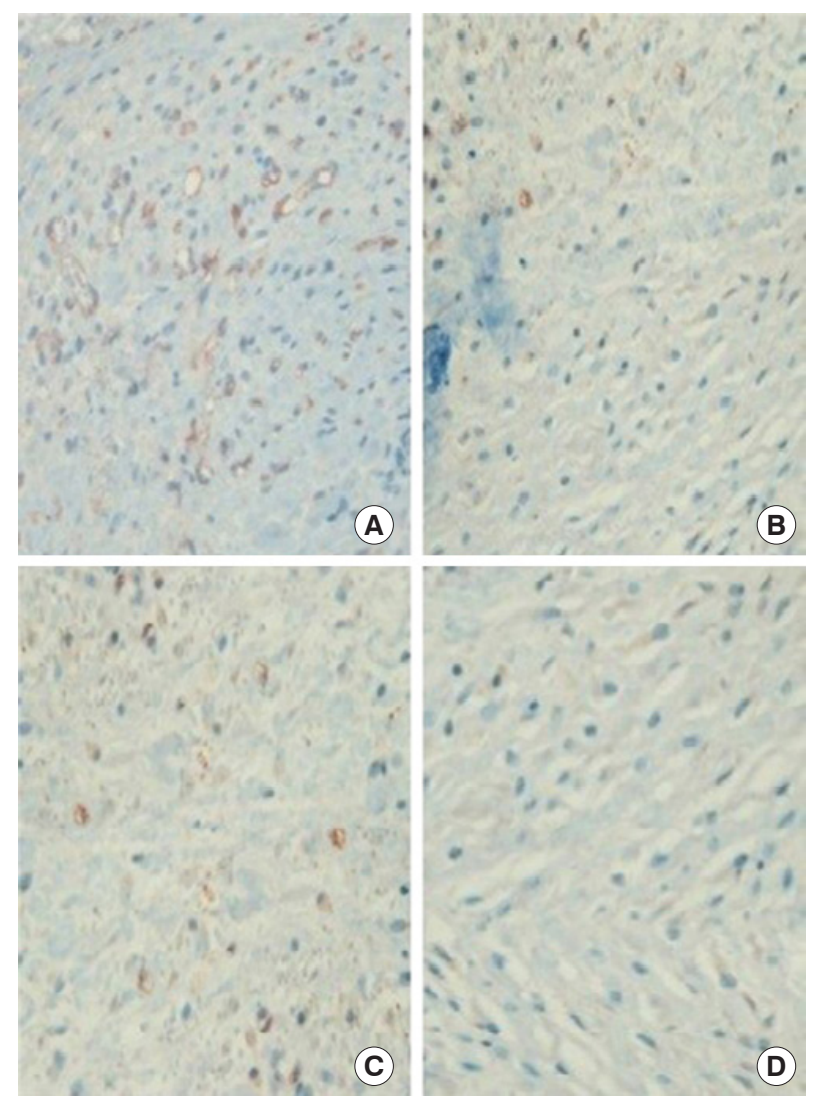

Fig. 8. Immunohistochemical staining of anti-rat CD31 antibody $(\times 400)$. The results showed more CD31-positive microvascular vessels (circular red-brown) in the FxCL+lidocaine group (A) than in the FxCL (B), lidocaine (C), and control groups (D). FxCL, fractional ablative carbon dioxide laser.

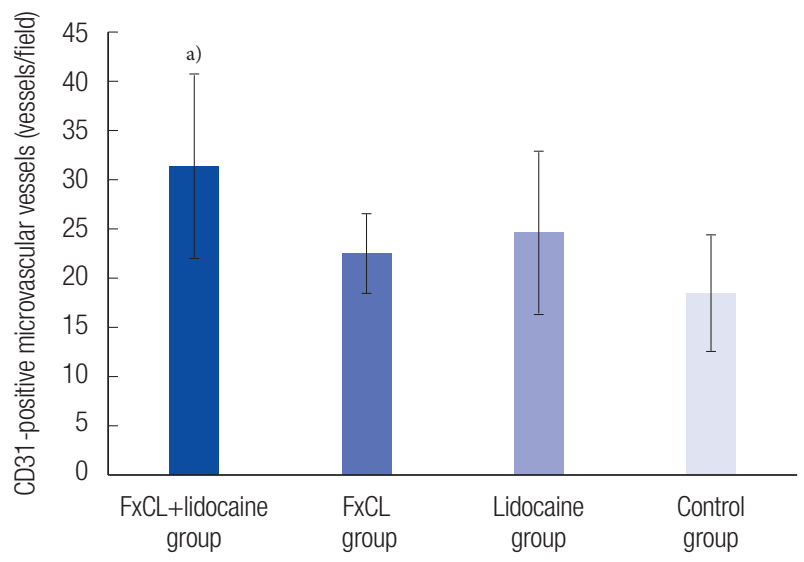

Fig. 9. CD31-positive microvascular vessels in the immunohistochemical evaluation. The number of CD31-positive microvascular vessels in the FxCL+lidocaine group was significantly higher than those in other groups (FxCL+lidocaine group, 31.38 \pm 9.36 vessels/field; FxCL group, $22.52 \pm 3.99$ vessels/field; lidocaine group, $24.66 \pm 8.25$ vessels/ field; control group, $18.45 \pm 5.89$ vessels/field). FxCL, fractional ablative carbon dioxide laser. ${ }^{\text {a) }} p<0.05$.

An ideal drug for improving flap survival would have the following characteristics: (1) clinical availability, (2) administra- 
tion easily, (3) high therapeutic index and safety, (4) reproducibility of effective results, (5) feasibility of postoperative treatment, (6) cost-effectiveness, (7) known mechanism of action, (8) established bioavailability, and (9) protective effect on necrosis [19]. No material to date meets all of these requirements. However, we believe that topical lidocaine is one of the closest to an ideal pharmacologic agent to enhance flap survival.

Lidocaine, a widely used amide-type local anesthetic, has been reported to enhance neoangiogenesis of random-pattern skin flaps [8]. When the surgeon decides to use lidocaine to improve flap survival, the administration route also has to be decided. Injection has the possibility of intravenous absorption, which may result in unintended higher concentration of the agent. Topical lidocaine, such as a cream or spray, is considered safer than injectable lidocaine for minimizing systemic adverse effects. Furthermore, such drugs can be easily administered postoperatively, even in an outpatient clinic or as bedside treatment, in clinical practice. However, topical lidocaine spray can be interrupted by the natural skin barrier. The therapeutic efficacy of topical drugs is related to their ability to reach target cells through the skin layer. The major rate-limiting step for percutaneous absorption is passage through the stratum corneum.

Reperfusion injury is an inflammatory process modulated by complex signaling mechanisms, which ultimately leads to cell death. As inflammation progresses, neutrophils accumulate. Neutrophil count could be used to predict flap injury [8]. Lidocaine blocks voltage-gated sodium channels in cell membranes of neurons and has been shown to improve cutaneous blood flow, inhibit platelet aggregation, and suppress neutrophil function. The present study demonstrated that neutrophil density was significantly lower in the FxCL+lidocaine group (27.20 \pm $4.24, p<0.001$ ) compared with control group.

The fractional ablative laser is a peculiar way that makes microscopic vertical holes on skin surface, each surrounded by a thin layer of ablated tissue, constituting microscopic ablation zones [13]. The laser leaves microscopic intervening areas of unexposed skin that can be remodeled without scarring. The laser facilitates penetration and distribution of topically applied drugs because the ablated laser holes extend into the dermis and act as channels for drug absorption [14].

Recently, low fluence setting of the fractional ablative laser, such as 2 or $3 \mathrm{~J} / \mathrm{cm}^{2}$, was reported to enhance topical drug delivery [20]. Fractional ablative laser therapy with low fluence has some advantages, including lesser pain and tissue damage and faster recovery than conventional fractional ablative laser therapy. In a pilot study, increasing the laser energy led to visible bleeding, edema and exudate in the treated areas. These factors might make it difficult for lidocaine to be absorbed into the skin against the hydrostatic/oncotic pressure pushing fluid out of the skin. In the histologic evaluation in this study, the stratum corneum was successfully disrupted by lower energy of $3 \mathrm{~J} / \mathrm{cm}^{2}$.

In this study, the FxCL group was necessary to determine the effect of the fractional ablative laser alone on flap survival. Based on our results, laser treatment can enhance survival of random-pattern skin flaps independently. Similarly, Prado et al. [21] reported the effect of low-level laser therapy, which could enhance random-pattern skin flap survival in rats. Low-power lasers have also been reported to have biostimulating effects, including neoangiogenesis, increased blood flow, and fibroblast proliferation. In the FxCL group, the flap received laser treatment only without pharmacologic agents. Although there were no significant differences, all parameters, including percentage of survival area, neutrophil count, vascular density, and VEGF/ CD31 expression, represented a more improved result compared with the control group. It is considered that increased vascular perfusion occurred in the FxCL group. Likewise, in the lidocaine group, most parameters demonstrated enhancement of flap survival. It is considered that the impact of lidocaine, including anti-inflammatory, antithrombotic, and ischemic-reperfusion injury prevention effects, could have produced the result in the lidocaine group without FxCL treatment.

To evaluate neoangiogenesis, vascular density in high-power fields and VEGF/CD31 expression were examined in this study. Although there was a significant difference in VEGF/CD31 expression in the FxCL+lidocaine group, the number of capillary vessels was not significantly different among the groups. There is a possibility of missing values or overestimation of the number of microvascular structures on $\mathrm{H} \& \mathrm{E}$ staining because a preexisting vessel could be considered as neoangiogenesis. Most importantly, the markedly enhanced effect of FxCL+lidocaine was significantly different from that of FxCL alone and lidocaine alone in all parameters. Thus, the neoangiogenetic and anti-inflammatory effects of lidocaine may be maximized with FxCL treatment.

The potential effect of FxCL and topical lidocaine spray on survival of random-pattern skin flaps in rats was investigated. This study showed the positive effect of FxCL on enhancement of random-pattern skin flap survival in rats with lidocaine. Therefore, FxCL with lidocaine spray can be considered a new therapeutic approach to enhance flap viability.

\section{NOTES}

\section{Conflict of interest}

No potential conflict of interest relevant to this article was reported. 


\section{Ethical approval}

The study was approved by the Institutional Animal Care and Use Committee of Yeungnam University (approval No. YUMC2015-011).

\section{ORCID}

Manki Choi https://orcid.org/0000-0003-2695-4139

Youngsoo Park https://orcid.org/0000-0001-5507-8049

Yong-Ha Kim https://orcid.org/0000-0002-1804-9086

Kyu Jin Chung https://orcid.org/0000-0001-6335-1818

\section{REFERENCES}

1. Yang M, Sheng L, Li H, Weng R, Li QF. Improvement of the skin flap survival with the bone marrow-derived mononuclear cells transplantation in a rat model. Microsurgery 2010;30:27581.

2. Davis RE, Wachholz JH, Jassir D, Perlyn CA, Agrama MH. Comparison of topical anti-ischemic agents in the salvage of failing random-pattern skin flaps in rats. Arch Facial Plast Surg 1999;1:27-32.

3. Kim CM, Oh JH, Jeon YR, Kang EH, Lew DH. Effects of human adipose-derived stem cells on the survival of rabbit ear composite grafts. Arch Plast Surg 2017;44:370-7.

4. Shalom A, Friedman T, Westreich M. The effect of postoperative aspirin on random pattern flaps in rats. Am Surg 2007;73: 1126-8.

5. Miyawaki T, Jackson IT, Bier UC, Andrus L, Williams F, Bradford $M$. The effect of capsaicin ointment on skin for the survival of a cutaneous flap. Eur J Plast Surg 2001;24:28-30.

6. Nakanishi Y, Nakajima T, Yoshimura Y, Okamoto Y, Yamada T. The transepidermal absorption of prostaglandin E1 as a topical ointment: an experimental study of application over a rat skin flap. Ann Plast Surg 1998;40:44-7.

7. Eskitascioglu T, Karaci S, Canoz O, Kilıc E, Gunay GK. The impact of lidocaine on flap survival following reperfusion injury. J Surg Res 2011;167:323-8.

8. Cao B, Wang L, Lin D, Cai L, Gao W. Effects of lidocaine on random skin flap survival in rats. Dermatol Surg 2015;41:53-8.

9. Ghali S, Knox KR, Verbesey J, Scarpidis U, Izadi K, Ganchi PA. Effects of lidocaine and epinephrine on cutaneous blood flow. J Plast Reconstr Aesthet Surg 2008;61:1226-31.

10. Huang GS, Lin TC, Wang JY, Ku CH, Ho ST, Li CY. Lidocaine priming reduces ADP-induced P-selectin expression and platelet-leukocyte aggregation. Acta Anaesthesiol Taiwan 2009; 47:56-61.

11. Kawasaki C, Kawasaki T, Ogata M, Sata T, Chaudry IH. Lidocaine enhances apoptosis and suppresses mitochondrial functions of human neutrophil in vitro. J Trauma 2010;68:401-8.

12. Guschlbauer M, Feige K, Geburek F, Hoppe S, Hopster K, Propsting MJ, et al. Effects of in vivo lidocaine administration at the time of ischemia and reperfusion on in vitro contractility of equine jejunal smooth muscle. Am J Vet Res 2011;72:144955.

13. Haedersdal M, Sakamoto FH, Farinelli WA, Doukas AG, Tam J, Anderson RR. Fractional CO(2) laser-assisted drug delivery. Lasers Surg Med 2010;42:113-22.

14. Lippert J, Smucler R, Vlk M. Fractional carbon dioxide laser improves nodular basal cell carcinoma treatment with photodynamic therapy with methyl 5-aminolevulinate. Dermatol Surg 2013;39:1202-8.

15. Waibel JS, Wulkan AJ, Shumaker PR. Treatment of hypertrophic scars using laser and laser assisted corticosteroid delivery. Lasers Surg Med 2013;45:135-40.

16. Bachhav YG, Heinrich A, Kalia YN. Using laser microporation to improve transdermal delivery of diclofenac: increasing bioavailability and the range of therapeutic applications. Eur J Pharm Biopharm 2011;78:408-14.

17. Chen X, Shah D, Kositratna G, Manstein D, Anderson RR, Wu MX. Facilitation of transcutaneous drug delivery and vaccine immunization by a safe laser technology. J Control Release 2012;159:43-51.

18. Oni G, Lequeux C, Cho MJ, Zhang D, Lazcano E, Brown SA, et al. Transdermal delivery of adipocyte-derived stem cells using a fractional ablative laser. Aesthet Surg J 2013;33:109-16.

19. Rohrich RJ, Cherry GW, Spira M. Enhancement of skin-flap survival using nitroglycerin ointment. Plast Reconstr Surg 1984;73:943-8.

20. Lee WR, Shen SC, Pai MH, Yang HH, Yuan CY, Fang JY. Fractional laser as a tool to enhance the skin permeation of 5-aminolevulinic acid with minimal skin disruption: a comparison with conventional erbium:YAG laser. J Control Release 2010; 145:124-33.

21. Prado RP, Pinfildi CE, Liebano RE, Hochman BS, Ferreira LM. Effect of application site of low-level laser therapy in random cutaneous flap viability in rats. Photomed Laser Surg 2009;27: 411-6. 